\title{
INFLATION IN A GENERAL REIONIZATION SCENARIO
}

\author{
STEFANIA PANDOLFI \\ ICRA and Universita' di Roma "La Sapienza", Ple. Aldo Moro 2, 00185, Rome, Italy \\ stefaniapandolfi@gmail.com
}

\begin{abstract}
Inflation predicts primordial scalar perturbations with a nearly scale-invariant spectrum and a spectral index approximately unity (the Harrison-Zel'dovich (HZ) spectrum). The first important step for inflationary cosmology is to check the consistency of the HZ primordial spectrum with current observations. Recent analyses have claimed that a $\mathrm{HZ}$ primordial spectrum is excluded at more than $99 \%$ c.l. Here we show that the HZ spectrum is only marginally disfavored if one considers a more general reionization scenario. Data from the Planck mission will settle the issue.
\end{abstract}

Keywords: Inflation, Reionization

\section{Introduction}

One usually models the dynamics of primordial inflation by the dynamics of a scalar field, know as the inflaton, evolving under the influence of a scalar potential. The scalar and tensor perturbation spectra produced by inflation depend upon the value of the inflaton potential during inflation and how rapidly the scalar field evolves during inflation. Observational information of the spectra can be translated into information about the inflaton potential, thus about physics far beyond the scales of the standard model of particle physics ${ }^{1}$. Cosmic microwave background (CMB) observables that map onto the the potential and its slope are 1) $n$, the spectral index of comoving scalar perturbations $P_{\mathcal{R}}: n-1=d \ln P_{\mathcal{R}} / d \ln k$, and 2) $r$, the ratio of tensor (gravitational wave) perturbations to $P_{\mathcal{R}}{ }^{2}$. Recent measurements by the Wilkinson Microwave Anisotropy Probe (WMAP) satellite seven-year mission ${ }^{3}$, combined with other experiments such as BOOMERanG ${ }^{4}, \mathrm{QUAD}^{5}, \mathrm{ACBAR}^{6}$, and $\mathrm{BICEP}^{7}$ have sharpened our knowledge of some key inflationary parameters. With regard to the dynamics of inflation, a hotly debated question is whether the case of $n=1$ is significantly excluded by current observations (see e.g., Refs. 3-13). The scalar spectral index, $n$, has been recently constrained to the value $n=0.963 \pm 0.014$ at $68 \%$ c.l. by the WMAP seven-year dataset (WMAP7) ${ }^{3}$, disfavoring the value of $n=1$ at about two standard deviations. A combined analysis with galaxy clustering data gives $n=0.963 \pm 0.012$ at $68 \%$ c.l., ruling out $n=1$ at more than three standard deviations ${ }^{3-13}$. Compelling evidence that $n \neq 1$ would be quite revealing for two reasons. First, it would provide an indication for the dynamical 
evolution of the expansion rate of the universe as perturbations are being produced. Secondly, in many models of slow-roll inflation the amplitude of the tensor perturbations are proportional to $|n-1|$. Thus, the larger the departure of $n$ from unity, the more likely tensor perturbations would be within observational reach. As it is well known, a scalar spectral index with an exact value of $n=1$ corresponds to the phenomenological model proposed by Harrison, Zel'dovich, and Peebles ${ }^{14 a}$. Observations pointing to $n$ exactly unity may indicate that the origin of cosmic perturbations lies in some unknown fundamental process and may not arise from inflation. It is well known from a large set of astrophysical observables that after primordial recombination (which occurred at a redshift of $z \sim 1100$ ) the universe "reionized" at a redshift $z>6$. Current constraints from CMB data assume a sudden and complete reionization at a redshift $z_{r}$. The electron ionization fraction $x_{e}(z)$ is parametrized by $z_{r}$ such that for $z \ll z_{r} x_{e}(z)=1\left(x_{e}(z)=1.08\right.$ for $z<3$ in order to take into account Helium recombination) and $x_{e}(z)=2 \times 10^{-4}$ for $z>z_{r}$, i.e., joining the value after primordial recombination with a smooth interpolation. However, the precise details of the reionization process are not very well known, and therefore the reionization history of the universe at those redshifts could have easily been very different. As already remarked in Refs. 17 and 18, the assumption of a more general reionization scheme could affect the cosmological constraints on $n$. In this paper we investigate the impact of the reionization history on the current constraints on $n$ in a more general reionization scenario. We show that the value of $n$, and its uncertainty, derived from CMB datasets are sensitive to how one models the reionization of the universe. In particular, the statement that $n=1$ is observationally excluded is very much weakened if one treats reionization in a general manner.

\section{Analysis and Results}

We adopt the method for parameterizing the reionization history developed in Ref. 17: it is based on principal components that provide a complete basis for describing the effects of reionization on large-scale $E$-mode polarization. Following Ref. 17, one can parametrize the reionization history as a free function of redshift by decomposing $x_{e}(z)$ into its principal components:

$$
x_{e}(z)=x_{e}^{f}(z)+\sum_{\mu} m_{\mu} S_{\mu}(z),
$$

where the principal components, $S_{\mu}(z)$, are the eigenfunctions of the Fisher matrix that describes the dependence of the polarization spectra on $x_{e}(z), m_{\mu}$ are the amplitudes of the principal components for a particular reionization history, and $x_{e}^{f}(z)$ is the WMAP fiducial model at which the Fisher matrix is computed and from which the principal components are obtained ${ }^{17}$. Hereafter we refer to this

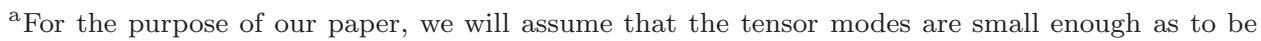
irrelevant. 
method as the MH (Mortonson-Hu) case. We have then modified the Boltzmann CAMB code ${ }^{19}$ incorporating the generalized reionization scenario and extracted cosmological parameters from current data using a Monte Carlo Markov Chain (MCMC) analysis based on the publicly available MCMC package cosmomc ${ }^{20}$. We consider here a flat $\Lambda$ CDM universe described by a set of cosmological parameters $\left\{\omega_{b}, \omega_{c}, \Theta_{s}, n, \log \left[10^{10} A_{s}\right]\right\}$. In one case we will also consider the possibility of a redshift-dependent dark energy component evolving with equation of state $w(z)=w_{0}+w_{z} z /(1+z)$. The extra parameters needed to describe the MH reionization are the five amplitudes of the eigenfunctions. Our basic data set is the seven-year WMAP data ${ }^{3}$ (temperature and polarization) and we also augment the WMAP7 data with the CMB datasets from BOOMERanG ${ }^{4}, \mathrm{QUAD}^{5}, \mathrm{ACBAR}^{6}$, and BICEP 7 (the " CMB All"' case) Finally we also consider, separately, the galaxy clustering results from LRG-7 of Ref. 21 and the baryonic acoustic oscillation (BAO) data from the same dataset, see Ref. 22. Our main results are reported in the following table. Notice that using the $\mathrm{MH}$ parametrization drastically alters the constraints on $n$. For the WMAP7 case alone, the HZ spectrum is not only in agreement with the data, but is also close to the best-fit value. When the remaining CMB experiments are included, the best-fit value of $n$ shifts to lower values, but it is still consistent at better than $95 \%$ c.l. with $n=1$. When information from galaxy clustering is included, a value of $n=1$ is excluded at about the $95 \%$ c.l., i.e., in a much less conclusive way than in the sudden case where $n=1$ is excluded at more than $99.7 \%$ c.l. (see e.g., Ref. 13). It is interesting to consider the constraints on the optical depth $\tau$, derived by integrating $x_{e}(z)$ up to $z=32$. Figure 1 shows the $68 \%$ and $95 \%$ c.l. constraints in the $n$ vs. $\tau$ plane arising from different datasets and assuming the $\mathrm{MH}$ reionization parametrization. As we can see the optical depth is always in the range $0.08-0.11$, slightly higher than in the standard analysis but consistent with several physical reionization models. Notice also that the HZ spectrum $(n=1)$ is perfectly compatible with WMAP7 (outer right contours), in two standard deviation in the case "CMB all' (middle contours) and only marginally excluded in the case "CMB All + BAO" (outer left contours). Moreover, considering a redshift-dependent equation of state weakens the geometrical probes and $\mathrm{HZ}$

\begin{tabular}{lcc}
\hline Dataset & $n(68 \%$ c.l. $)$ & $95 \%$ c.l. \\
\hline \hline WMAP7 & $0.993 \pm 0.023$ & $n \leq 1.042$ \\
CMB All & $0.975 \pm 0.017$ & $n \leq 1.011$ \\
CMB All+LRG-7 & $0.966 \pm 0.014$ & $n \leq 0.994$ \\
CMB All+BAO & $0.965 \pm 0.014$ & $n \leq 0.995$ \\
CMB All+BAO+w(z) & $0.985 \pm 0.018$ & $n \leq 1.025$ \\
WMAP7 & $0.977 \pm 0.018$ & $n \leq 1.01$ \\
CMB All & $0.963 \pm 0.015$ & $n \leq 0.998$ \\
\hline \hline
\end{tabular}




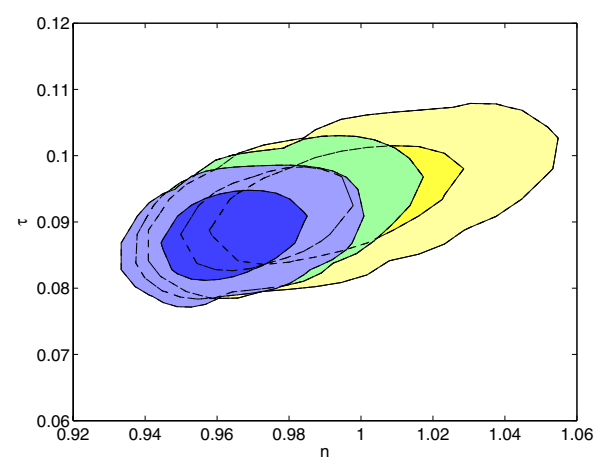

Fig. 1. Contours of the $68 \%$ and $95 \%$ c.l. constraints in the $n$ vs $\tau$ plane for different datasets. The contours regions come from a generalized reionization scenario using (contours from right to left) the MH approach from WMAP-7 data, "CMB all", "CMB All+BAO". WARNING: see if it clear what I mean, I cannot put it in $\mathrm{B} / \mathrm{W}$ but $\mathrm{I}$ changed text and caption in order to make it clear without referring to colours

is again inside the $68 \%$ confidence level for the $\mathrm{CMB}+\mathrm{BAO}$ case. It is important to investigate if a more general reionization scenario with the assumption of $n=1$ could provide a viable cosmology, i.e., if the value of cosmological parameters such as the baryon density or the age of the universe are compatible with complementary cosmological information from Big Bang Nucleosythesis and age constraints. It has been shown, for example, in Ref. ${ }^{13}$ that the assumption of an HZ spectrum in the standard analysis with sudden reionization gives a baryon abundance that is at odds with current bounds from BBN. We have therefore performed an analysis using WMAP7 and assuming $n=1$ for the MH reionization scenario, and the results are fully consistent if compared with those obtained with other independent cosmological observables. For instance, we obtain that $\Omega_{b} h^{2}=0.0234 \pm 0.0004, H_{0}$ $\left[\mathrm{km} \mathrm{s}^{-1} \mathrm{Mpc}^{-1}\right]=74.2 \pm 2.1$ and $\Omega_{\Lambda}=0.765 \pm 0.022$. Current Big Bang Nucleosynthesis constraints from observations of Deuterium abundances provide a constraint of $\Omega_{b} h^{2}=0.0213 \pm 0.002$ at $95 \%$ c.l. ${ }^{23}$, which is compatible with the WMAP7 constraint assuming HZ. The constraints on $H_{0}$ are also well in agreement with the recent HST determination $h=0.748 \pm 0.036$ at $68 \%$ c.l. ${ }^{24}$. In conclusion, the details of the reionization processes in the late universe are not very well known. In the absence of a precise, full redshift evolution description of the ionization fraction during the reionization period, a simple parametrization, with a single parameter $z_{r}$, has become the standard reionization scheme in numerical analyses. However, more general reionization scenarios are certainly plausible and their impact on the cosmological constraints should be carefully explored. For example, processes of dark matter annihilation as in Ref. ${ }^{25}$ could easily be present. In this paper we have investigated the stability of the CMB constraints on $n$ in generalized reionization scenarios. We have found that a more general treatment of reionization drastically weakens current $\mathrm{CMB}$ constraints on $n$. Therefore, current data do not yet rule out an $\mathrm{HZ}$ spectrum in a conclusive way. 


\section{References}

1. J. E. Lidsey, A. R. Liddle, E. W. Kolb, E. J. Copeland, T. Barreiro and M. Abney, Rev. Mod. Phys. 69, 373 (1997).

2. S. Dodelson, W. H. Kinney and E. W. Kolb, Phys. Rev. D 56, 3207 (1997).

3. E. Komatsu et al., arXiv:1001.4538 [astro-ph.CO]; D. Larson et al., arXiv:1001.4635 [astro-ph.CO].

4. W. C. Jones et al., Astrophys. J. 647, 823 (2006); F. Piacentini et al., Astrophys. J. 647, 813 (2006), Astrophys. J. 647, 833 (2006).

5. M. L. Brown et al. [QUaD collaboration], Astrophys. J. 705, 978 (2009).

6. C. L. Reichardt et al., Astrophys. J. 694 (2009) 1200.

7. H. C. Chiang et al., arXiv:0906.1181 [astro-ph.CO].

8. W. H. Kinney, E. W. Kolb, A. Melchiorri, and A. Riotto, Phys. Rev. D 74, 023502 (2006); W. H. Kinney, E. W. Kolb, A. Melchiorri, and A. Riotto, Phys. Rev. D 78 (2008) 087302.

9. D. Parkinson, P. Mukherjee, and A. R. Liddle, Phys. Rev. D 73, 123523 (2006).

10. J. Hamann, S. Hannestad, M. S. Sloth, and Y. Y. Y. Wong, Phys. Rev. D 75, 023522 (2007).

11. M. Bridges, A. N. Lasenby, and M. P. Hobson, arXiv:astro-ph/0607404.

12. J. Martin and C. Ringeval, JCAP 0608, 009 (2006).

13. F. Finelli, J. Hamann, S. M. Leach, and J. Lesgourgues, arXiv:0912.0522 [astroph.CO].

14. E. R. Harrison, Phys. Rev. D 1, 2726 (1970); Y. B. Zel'dovich, Mon. Not. Roy. Astron. Soc. 160, (1972), P. J. E. Peebles and J. T. Yu Astrophys. J. 162, 815 (1970).

15. A. Vallinotto, E. J. Copeland, E. W. Kolb, A. R. Liddle, and D. A. Steer, Phys. Rev. D 69, 103519 (2004).

16. A. A. Starobinsky, JETP Lett. 82, 169 (2005) [Pisma Zh. Eksp. Teor. Fiz. 82, 187 (2005)].

17. M. J. Mortonson and W. Hu, Astrophys. J. 686, L53 (2008).

18. A. Lewis, J. Weller, and R. Battye, Mon. Not. Roy. Astron. Soc. 373, 561 (2006).

19. A. Lewis, A. Challinor, and A. Lasenby, Astrophys. J. 538 (2000) 473.

20. A. Lewis and S. Bridle, Phys. Rev. D 66, 103511 (2002).

21. B. A. Reid et al., arXiv:0907.1659 [astro-ph.CO].

22. W. J. Percival et al., arXiv:0907.1660 [astro-ph.CO].

23. M. Pettini, B. J. Zych, M. T. Murphy, A. Lewis, and C. C. Steidel, Mon. Not. Roy. Astron. Soc. 391, 1499 (2008).

24. A. G. Riess et al., Astrophys. J. Suppl. 183, 109 (2009).

25. S. Galli, F. Iocco, G. Bertone and A. Melchiorri, Phys. Rev. D 80 (2009) 023505.

26. S. Galli, R. Bean, A. Melchiorri and J. Silk, Phys. Rev. D 78 (2008) 063532.

27. R. Bowen, S. H. Hansen, A. Melchiorri, J. Silk and R. Trotta, Mon. Not. Roy. Astron. Soc. 334, 760 (2002); J. Hamann, S. Hannestad, J. Lesgourgues, C. Rampf and Y. Y. Y. Wong, arXiv:1003.3999 [astro-ph.CO].

28. [Planck Collaboration], arXiv:astro-ph/0604069. 